\title{
An ethnobotanical note on wild edible plants of Upper Eastern Himalaya, India
}

\section{G. Murtem ${ }^{1}$ and Pradeep Chaudhry ${ }^{2}$}

${ }^{1}$ State Forest Research Institute. P.O. Van Vihar, Chimpu, Itanagar-791113, Arunachal Pradesh, India.

2Indian Institute of Forest Management. Nehru Nagar, Bhopal-462003, India. Email: pradeepifs@yahoo.com.

\begin{abstract}
The present article deals with the documentation of tribal knowledge on wild edible plants in Upper Eastern Himalayan Region of India. In total, 269 plant species belonging to 77 families were recorded in the study area of Upper Subansiri District of Arunachal Pradesh. Dioscoreaceae, Rutaceae, Cucurbitaceae and Arecaceae were the dominant families with 16, 13, 12 and 11 plant species, respectively, while Moraceae and Asteraceae followed with 10 species each. Four major life forms were trees, shrubs, herbs and climbers. Trees made the highest proportion of the edible species (81) followed by shrubs (74), herbs (71) and climbers (37). Availability of some of these species might be a serious constraint in near future due to various anthropogenic factors. Need for undertaking vigorous environmental awareness campaign among local tribes has been stressed upon in the article for conserving this valuable biological resource of Eastern Himalayan region of India.
\end{abstract}

Keywords: Wild edible plants; Hill Miri Tribe; Nyishi Tribe; Tagin Tribe; Galo Tribe; Arunachal Pradesh.

\section{Introduction}

Arunachal Pradesh is one of the biodiversity rich states of India in terms of flora and fauna. The state is the largest one among all north eastern states of India situated in the lap of eastern Himalayan region of the country. Varied climatic, topographical and soil conditions favour high species richness and support different types of forests. Broad forest types include tropical moist deciduous, tropical semi
Received

March 22, 2016

Accepted May 11, 2016

Released June 30, 2016

Open Acess Full Text Article

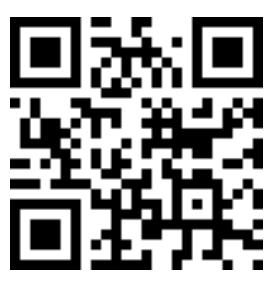

\section{ORCIID}

- 0000-0002-8375-7118 G. Murtem

이 0000-0003-3792-1199 Pradeep Chaudhry evergreen, tropical evergreen, subtropical, temperate and alpine forests. Recorded number of flowering plant species $(>5,000)$ are the highest among all North Eastern states (Hegde, 2000). The state is inhabited by 28 major tribes and 110 sub tribes (Khongsai et al., 2011) who depend immensely on forest flora and fauna for their day to day needs and requirements. It is estimated that, in India about 800 species are consumed as food plants whereas the corresponding figure for north east (NE) 
India is around 300. Almost all the edible plants of NE region are represented in Arunachal Pradesh (Arora, 1981; Haridasan et al., 1990).

Many research studies have been conducted on the diversity and traditional uses of wild plants from Arunachal Pradesh state, particularly on medicinal plants (Pal, 1984, 1992; Rawat et al., 1996, 1998; Tag and Das, 2004; Shankar et al., 2008, 2011, 2016; Goswami et al., 2009; Khongsai et al., 2011). Although much has been reported on ethnomedicinal aspects of plants of the region, little has been reported about the wild edible plants of the state. Keeping this aspect in view, a study was conducted as an attempt to explore and identify the wild edible plant resources and indigenous traditional knowledge about their utilization in the Upper Subansiri district of Arunachal Pradesh during 20102012 by the State Forest Research Institute, Itanagar, Arunachal Pradesh. The Upper Subansiri District of Arunachal Pradesh lies in the central part of the state in between $28.5^{\circ}$ and $28.25^{\circ}$ latitudes $\mathrm{N}$ and $93.15^{\circ}$ and $94.20^{\circ}$ longitudes E covering a geographical area on $7,032 \mathrm{~km}^{2}$ (Figure 1). The high mountain region near northern boundary of the district is generally cold as remain covered with snow almost throughout the year. The plain areas in foothills are intersected by number of water bodies mainly streams and rivers in the lap of forests (Goswami et al., 2009). The district is inhabited by three major tribes namely Tagin, Hill Miri (now Nyishi) and Galo.

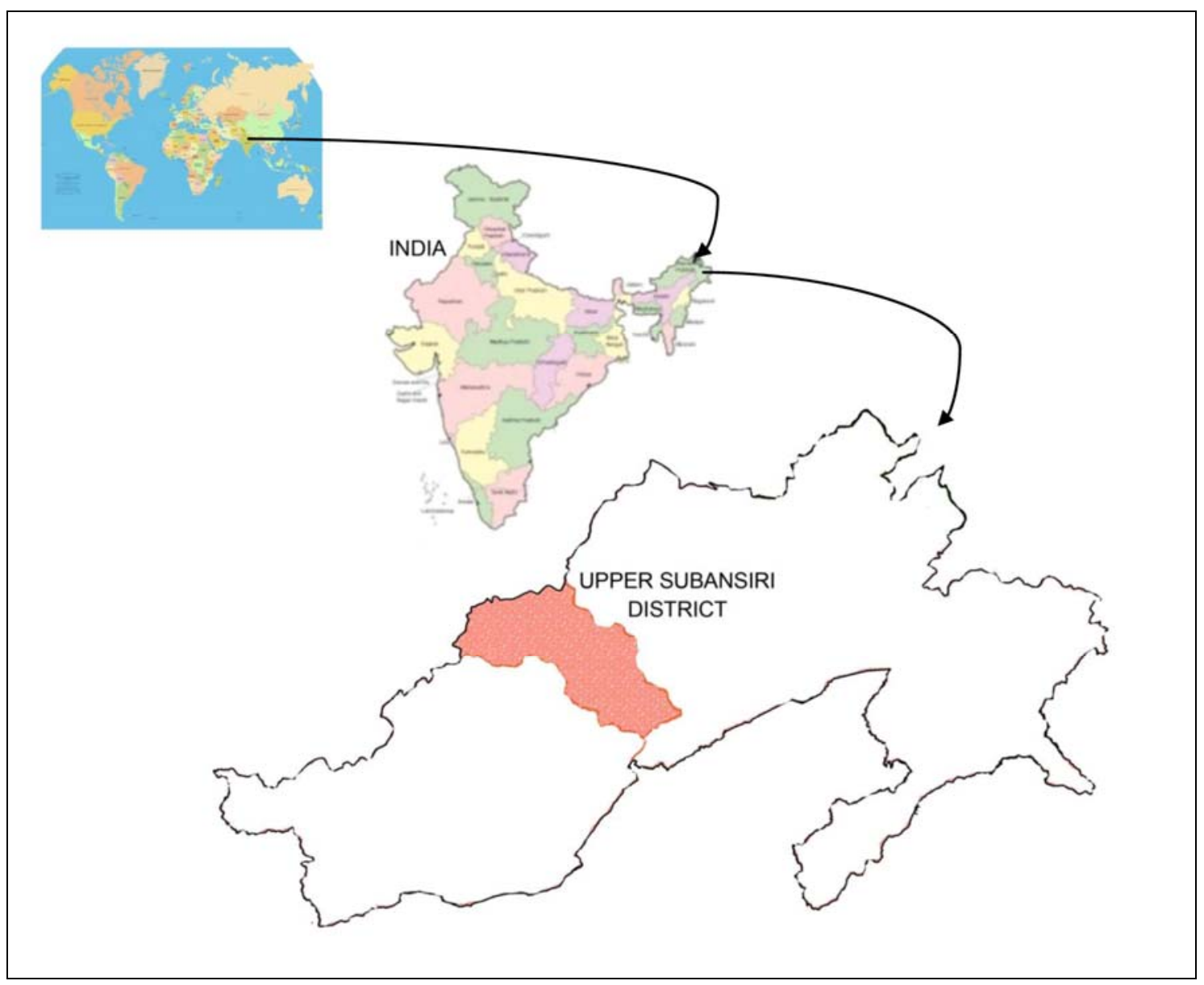

Figure 1. Location map of Upper Subansiri district, Arunachal Pradesh, India. Source: Government of Arunachal Pradesh, India. Available from: <http://www.arunachalpradesh.gov.in>. 


\section{Methodology}

The ethnobotanical information was collected by field works/survey among aboriginal community, by study with herbaria and museum, by study of rituals, myths and folklores and through folk market survey. Tribal markets or weekly haats (local market) were also visited to study the plants sold there. Maps prepared by the Directorate of Economics and Statistics, Government of Arunachal Pradesh were also consulted for identification of approaching routes and location of the select villages. The villages inhabited by the tribesmen were selected randomly for each tribe for the purpose of survey (Table 1).

Table 1. Villages covered during study in Upper Subansiri District.

\begin{tabular}{llll}
\hline S. No. & Name of the Tribe & Name of the village & Name of the circle \\
\hline 1 & Nyshi & i) Godak & Raga \\
& & ii) Muri Mugli & Puchi-Geko \\
& iii) Babla & Puchi-Geko \\
& iv) Don & Daporijo \\
& v) Dakpe & Daporijo \\
vi) Ligu & Daporijo \\
& vii) Niji & Daporijo \\
& viii) Mite & Daporijo \\
& ix) Jigi & Daporijo \\
& i) Sippi & Giba \\
& ii) Manga & Giba \\
& iii) Talihia & Taliha \\
& iv) Siyum & Siyum \\
& v) Nacho & Nacho \\
& vi) Limiking & Limiking \\
& vagin & vii) Orak & Limiking \\
& viii) Reddi & Limiking \\
& ix) Taksing & Taksing \\
& i) Dumporijo & Dumporijo \\
& ii) Pakka & Dumporijo \\
& iii) Haji & Baririjo \\
& iv) Maro & Baririjo \\
& v) Dula & Baririjo \\
& vi) Tapi & Baririjo \\
& vii) Tashi Doni & Baririjo \\
\hline 3 & &
\end{tabular}

\section{Results and discussion}

The present investigation comprising of 269 species of plants belonging to 77 families and details like botanical name, local name, family, parts used have been enumerated in Table 2 . These species are used by the tribes as wild fruits, vegetables, beverages or in other preparations. 
Table 2. Wild edible plants used by tribes of Upper Subansiri District.

\begin{tabular}{|c|c|c|c|c|c|}
\hline S. No. & Botanical name & Local name & Family & Habit & Uses \\
\hline 1 & $\begin{array}{l}\text { Griffithia fusca } \\
\text { Maingrey ex King. }\end{array}$ & Tok Schein & Annonaceae & Tree & Fruits are edible \\
\hline 2 & $\begin{array}{l}\text { Cissampelos pareira L. } \\
\text { Var.Linsuta } \\
\text { (Buch.Hami ex DC) }\end{array}$ & Tabo & Menispermaceae & Shrub & Fruits are edible \\
\hline 3 & Limacia oblonga Miers. & Titmilie & Menispermaceae & Shrub & Fruits are edible \\
\hline 4 & $\begin{array}{l}\text { Berberis asiatica Roxb. } \\
\text { ex DC }\end{array}$ & Tge & Berberidaceae & Shrub & Fruits are edible \\
\hline 5 & $\begin{array}{l}\text { Berberis wallichiana } \\
\text { DC }\end{array}$ & Tipetere & Berberidaceae & Shrub & Fruits are edible \\
\hline 6 & $\begin{array}{l}\text { Mohonia acanthifolia } \\
\text { G. Don. }\end{array}$ & Taming As & Berberidaceae & Shrub & Fruits are edible \\
\hline 7 & $\begin{array}{l}\text { Brassica campestris } \\
\text { Linn. }\end{array}$ & Horyo & Brassicaceae & Herb & $\begin{array}{l}\text { Leaves are used } \\
\text { as vegetable }\end{array}$ \\
\hline 8 & $\begin{array}{l}\text { Brassica juncea L. var. } \\
\text { cuneifolia } \\
\text { Kitampura }\end{array}$ & Goyu & Brassicaceae & Herb & $\begin{array}{lr}\text { Leaves } & \text { are } \\
\text { cooked } & \text { as } \\
\text { vegetable } & \end{array}$ \\
\hline 9 & $\begin{array}{l}\text { Cardamine hirusta } \\
\text { Linn. }\end{array}$ & Goj Ao & Brassicaceae & Herb & $\begin{array}{l}\text { Leaves are used } \\
\text { as vegetable }\end{array}$ \\
\hline 10 & suaveolens & Tasser tere & Cappaaridaceae & Climber & Fruits are edible \\
\hline & $\begin{array}{l}\text { (Roxb.) Pierre Syn. } \\
\text { Roydosia } \\
\text { Roxb. }\end{array}$ & & & & \\
\hline 11 & $\begin{array}{l}\text { Gypsophila cerastioides } \\
\text { D.Don. }\end{array}$ & Geeda Ao & Caryophylaceae & Herb & $\begin{array}{l}\text { Whole plant is } \\
\text { used as vegetable }\end{array}$ \\
\hline 12 & $\begin{array}{l}\text { Portulaca oleracea } \\
\text { Linn. }\end{array}$ & Tadar Ao & Portulacaceae & Herb & $\begin{array}{l}\text { Whole plant is } \\
\text { used as vegetable }\end{array}$ \\
\hline 13 & $\begin{array}{l}\text { Garcinia pedunculata } \\
\text { Roxb. }\end{array}$ & Mibia & Clusiaceae & Tree & Fruits are edible \\
\hline 14 & $\begin{array}{l}\text { Camellia sinensis (L.) } \\
\text { O. Ktze. Syn. Thea } \\
\text { sinensis L. }\end{array}$ & Saa Schein & Theaceae & Shrub & $\begin{array}{l}\text { Leaf extract is } \\
\text { used as beverage }\end{array}$ \\
\hline 15 & $\begin{array}{l}\text { Saurauia armata Kurz. } \\
\text { Syn. Saurauia cerea } \\
\text { Griff. }\end{array}$ & Hero & Saurauiaceae & Tree & Fruits are edible \\
\hline 16 & $\begin{array}{l}\text { Hibiscus esculentus } \\
\text { Linn. }\end{array}$ & Bhindi & Malvaceae & Shrub & Fruits are edible \\
\hline 17 & Bombax ceiba Linn. & $\begin{array}{l}\text { Semmul } \\
\text { Schein }\end{array}$ & Bombacaceae & Tree & $\begin{array}{l}\text { Flower buds are } \\
\text { used as vegetable }\end{array}$ \\
\hline 18 & Sterculia guttata Roxb. & $\begin{array}{l}\text { Tattem Belom, } \\
\text { Takom Belom }\end{array}$ & Sterculiaceae & Tree & $\begin{array}{lr}\text { Immature } & \text { seeds } \\
\text { eaten } & \text { by } \\
\text { removing } & \\
\text { pericarp } & \end{array}$ \\
\hline 19 & Grewia serrulata DC. & Litik & Tiliaceae & Tree & Fruits are edible \\
\hline 20 & $\begin{array}{l}\text { Eleocarpus floribundus } \\
\text { Blume. }\end{array}$ & Jolphai Schein & Eleocarpaceae & Tree & $\begin{array}{l}\text { Fruits are edible } \\
\text { both raw, cooked }\end{array}$ \\
\hline 21 & $\begin{array}{l}\text { Eleocarpus sphaerius } \\
\text { (Gaertn.) K.Schum. }\end{array}$ & Bhagwan tasse & Eleocarpaceae & Tree & $\begin{array}{l}\text { Young immature } \\
\text { fruits are edible }\end{array}$ \\
\hline 22 & $\begin{array}{l}\text { Oxalis acetosella Ls. } \\
\text { Syn. O. griffithi Edgew. }\end{array}$ & Pak Huku & Oxalidaceae & Herb & $\begin{array}{l}\text { Whole plant is } \\
\text { eaten raw as well } \\
\text { as eaten cooked }\end{array}$ \\
\hline
\end{tabular}


Table 2. Continued.

\begin{tabular}{|c|c|c|c|c|c|}
\hline S. No. & Botanical name & Local name & Family & Habit & Uses \\
\hline 23 & $\begin{array}{l}\text { Oxalis } \quad \text { corniculata } \\
\text { Linn. }\end{array}$ & -do- & Oxalidaceae & Herb & $\begin{array}{l}\text { Whole plant is } \\
\text { edible }\end{array}$ \\
\hline 24 & $\begin{array}{l}\text { Oxalis debilis H.B.K. } \\
\text { Var. Corymbosa (DC.) } \\
\text { Lourt. }\end{array}$ & -do- & Oxalidaceae & Herb & $\begin{array}{l}\text { Whole plant is } \\
\text { eaten for sour } \\
\text { taste }\end{array}$ \\
\hline 25 & $\begin{array}{l}\text { Impatiens racemosa } \\
\text { DC. }\end{array}$ & Yamchu & Balsaminaceae & Herb & $\begin{array}{l}\text { Leaves are used } \\
\text { as vegetable }\end{array}$ \\
\hline 26 & $\begin{array}{l}\text { Aegle marmelos (L.) } \\
\text { Correa. }\end{array}$ & Bhel & Rutaceae & Tree & Fruits are edible \\
\hline 27 & Citrus decumana Linn. & Sepen Nane & Rutaceae & Tree & Fruits are edible \\
\hline 28 & Citrus excavate Burm. & $\begin{array}{l}\text { Sepen Nane } \\
\text { Anye }\end{array}$ & Rutaceae & Tree & Fruits are eaten \\
\hline 29 & $\begin{array}{l}\text { Citrus limeon (Linn.) } \\
\text { Bl. }\end{array}$ & Nimbu & Rutaceae & $\begin{array}{l}\text { Small } \\
\text { tree }\end{array}$ & Fruits are edible \\
\hline 30 & Citrus limetta Risso. & Sepen & Rutaceae & Shrub & Fruits are edible \\
\hline 31 & Citrus medica Linn. & Nanya Sepen & Rutaceae & Shrub & Fruits are edible \\
\hline 32 & $\begin{array}{l}\text { Murraya koenigii (L.) } \\
\text { Spreng. }\end{array}$ & $\begin{array}{l}\text { Nangken } \\
\text { Nyibumturum }\end{array}$ & Rutaceae & Shrub & $\begin{array}{l}\text { Leaves used as } \\
\text { flavouring agent }\end{array}$ \\
\hline 33 & $\begin{array}{l}\text { Murraya } \quad \text { paniculata } \\
\text { (L.) Jack. }\end{array}$ & Nyibumturum & Rutaceae & Tree & $\begin{array}{l}\text { Ripened fruits } \\
\text { and leaves edible }\end{array}$ \\
\hline 34 & $\begin{array}{l}\text { Toddalia aculeate Pers. } \\
\text { Syn.T.asiatica } \\
\text { Lamk. }\end{array}$ & Tiktak Shein & Rutaceae & Shrub & Fruits are edible \\
\hline 35 & $\begin{array}{l}\text { Zanthoxylum } \\
\text { acanthopodium Edgew. }\end{array}$ & Honyor & Rutaceae & Shrub & $\begin{array}{lr}\text { Young leaves } \\
\text { eaten as } \\
\text { vegetable, raw } \\
\text { fruits also eaten }\end{array}$ \\
\hline 36 & $\begin{array}{l}\text { Zanthoxylum aramatum } \\
\text { DC Syn. Z. alatum } \\
\text { Roxb. }\end{array}$ & Honyar & Rutaceae & $\begin{array}{l}\text { Small } \\
\text { tree }\end{array}$ & $\begin{array}{l}\text { Young leaves } \\
\text { and fruits are } \\
\text { eaten }\end{array}$ \\
\hline 37 & $\begin{array}{l}\text { Zanthoxylum } \\
\text { oxyphyllum Edgew. }\end{array}$ & Honyor & Rutaceae & $\begin{array}{l}\text { Climber } \\
\text { shrub }\end{array}$ & $\begin{array}{l}\text { Leaves used as } \\
\text { vegetable }\end{array}$ \\
\hline 38 & $\begin{array}{l}\text { Zanthoxylum rhesta } \\
\text { (Roxb.) DC. }\end{array}$ & Honyor & Rutaceae & Shrub & $\begin{array}{l}\text { Leaves used as } \\
\text { vegetable }\end{array}$ \\
\hline 39 & $\begin{array}{l}\text { Canarium } \quad \text { strictum } \\
\text { Roxb. }\end{array}$ & Schellum & Burseraceae & Tree & Fruits are edible \\
\hline 40 & $\begin{array}{l}\text { Berchemia floribunda } \\
\text { (Wall.) Brongn. }\end{array}$ & Year Ao & Rhamnaceae & Shrub & $\begin{array}{l}\text { Leaves are used } \\
\text { as vegetable }\end{array}$ \\
\hline 41 & $\begin{array}{l}\text { Rhamnus nepalensis } \\
\text { (Wallich) Lawson }\end{array}$ & Biringa schein & Rhamnaceae & $\begin{array}{l}\text { Small } \\
\text { tree }\end{array}$ & Fruits are edible \\
\hline 42 & $\begin{array}{l}\text { Ziziphus mauritiana } \\
\text { Lam. Syn. } Z \text {. jujube } \\
\text { Lam. }\end{array}$ & Bogori & Rhamnaceae & $\begin{array}{l}\text { Small } \\
\text { tree }\end{array}$ & Fruits are edible \\
\hline 43 & Ziziphus rugosa Lamk. & Bogori & Rhamnaceae & Shrub & Fruits are edible \\
\hline 44 & Cissus repens Lamk. & - & Vitaceae & Climber & Fruits are edible \\
\hline 45 & $\begin{array}{l}\text { Leea indica (Burm.f.) } \\
\text { Merr. }\end{array}$ & $\begin{array}{l}\text { Demborang } \\
\text { Sagne }\end{array}$ & Leeaceae & $\begin{array}{l}\text { Small } \\
\text { tree }\end{array}$ & Fruits are edible \\
\hline 46 & $\begin{array}{l}\text { Litchi chinensis Sonnar. } \\
\text { Syn. Nephelium litchi } \\
\text { (Lour.) }\end{array}$ & Lisu, Litchi & Sapindaceae & Tree & Fruits are edible \\
\hline 47 & $\begin{array}{l}\text { Turpinia } \quad \text { pomifera } \\
\text { (Roxb.) DC. }\end{array}$ & Takre-Schein & Staphyleaceae & Tree & Fruits are edible \\
\hline 48 & Mangifera indica Roxb. & Toggu & Anacardiaceae & Tree & Fruits are edible \\
\hline
\end{tabular}


Table 2. Continued.

\begin{tabular}{|c|c|c|c|c|c|}
\hline S. No. & Botanical name & Local name & Family & Habit & Uses \\
\hline 49 & $\begin{array}{l}\text { Mangifera sylvatica } \\
\text { Roxb. }\end{array}$ & Motum Toggu & Anacardiaceae & Tree & Fruits are edible \\
\hline 50 & $\begin{array}{l}\text { Pegia nitida Colebr. } \\
\text { Syn. Tapiniria hirusta }\end{array}$ & Redin & Anacardiaceae & Shrub & Fruits are edible \\
\hline 51 & $\begin{array}{l}\text { Spondias axillaries } \\
\text { Roxb. }\end{array}$ & Belam Schein & Anacardiaceae & Tree & Fruits are edible \\
\hline 52 & $\begin{array}{l}\text { Spondias pinnata } \\
\text { (Linn.f.) Kurz. Syn. S. } \\
\text { mangifera Willd. }\end{array}$ & Tanya Schein & Anacardiaceae & Tree & $\begin{array}{l}\text { Young leaves are } \\
\text { used as } \\
\text { vegetable. } \\
\text { Ripened fruits } \\
\text { are also edible }\end{array}$ \\
\hline 53 & $\begin{array}{l}\text { Moringa oleifera Lamk. } \\
\text { Syn. M. pterygos perma }\end{array}$ & Saajna & Moringaceae & Tree & $\begin{array}{l}\text { Tender capsules } \\
\text { and young leaves }\end{array}$ \\
\hline & Gaertn, & & & & $\begin{array}{l}\text { are used as } \\
\text { vegetable }\end{array}$ \\
\hline 54 & $\begin{array}{l}\text { Cajanus cajan (L.) Syn. } \\
\text { C. indicus Spring }\end{array}$ & Daal & Fabaceae & Shrub & $\begin{array}{l}\text { Seeds are eaten } \\
\text { in cooked form }\end{array}$ \\
\hline 55 & $\begin{array}{l}\text { Canavalia insiformis } \\
\text { (L.) DC. Syn. C. C. } \\
\text { gladiata (Jacq.) DC. }\end{array}$ & Hepetepei & Fabaceae & Climber & Fruits are eaten \\
\hline 56 & $\begin{array}{lr}\text { Codariocalyx } & \text { motorius } \\
\text { (Houtt.) } & \text { Syn. } \\
\text { Desmodium } & \text { gyrans } \\
\text { (L.f.) DC. } & \end{array}$ & Sha Dach & Fabaceae & Shrub & $\begin{array}{l}\text { Leaves are } \\
\text { boiled in water } \\
\text { and dried, } \\
\text { thereafter used as } \\
\text { tea leaves }\end{array}$ \\
\hline 57 & Erythrina stricta Roxb. & Tagek Schein & Fabaceae & Tree & $\begin{array}{l}\text { Young tender } \\
\text { leaves are used } \\
\text { as vegetable }\end{array}$ \\
\hline 58 & 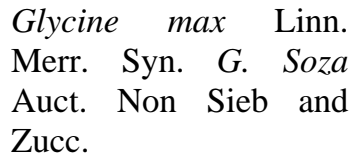 & Peayak & Fabaceae & Herb & $\begin{array}{l}\text { Seeds are } \\
\text { fermented and } \\
\text { eaten }\end{array}$ \\
\hline 59 & Lathyrus sativus Linn. & Emtor & Fabaceae & Herb & $\begin{array}{l}\text { Seeds and young } \\
\text { leaves eaten as } \\
\text { vegetable }\end{array}$ \\
\hline 60 & $\begin{array}{l}\text { Phaseolus } \\
\text { (Beans) }\end{array}$ & Paren & Fabaceae & Climber & $\begin{array}{l}\text { Pods are used as } \\
\text { vegetable }\end{array}$ \\
\hline 61 & $\begin{array}{l}\text { Bauhania purpurea } \\
\text { Linn. }\end{array}$ & Pacham & Caesalpiniaceae & Tree & $\begin{array}{l}\text { Young leaves } \\
\text { and flowers are } \\
\text { used as vegetable }\end{array}$ \\
\hline 62 & $\begin{array}{l}\text { Bauhania variegata } \\
\text { Linn. }\end{array}$ & Pachem & Caesalpiniaceae & Tree & $\begin{array}{l}\text { Young leaves } \\
\text { and flowers are } \\
\text { used as vegetable }\end{array}$ \\
\hline \multirow[t]{2}{*}{63} & Bauhania & Pachem & Caesalpiniaceae & Climber & Tender leaves \\
\hline & $\begin{array}{l}\text { Macbr. Syn. } \quad \text { B. } \\
\text { macrostachya } \\
\text { Beng. }\end{array}$ & & & & $\begin{array}{l}\text { are used as } \\
\text { vegetable }\end{array}$ \\
\hline 64 & $\begin{array}{l}\text { Tamarindus } \\
\text { Linn. }\end{array}$ & Khosuk naan & Caesalpiniaceae & Tree & $\begin{array}{l}\text { Fruit pulp is } \\
\text { eaten raw, used } \\
\text { in curries }\end{array}$ \\
\hline 65 & $\begin{array}{l}\text { Mitracarpus } \\
\text { verticillatus Schum. \& } \\
\text { Thom. }\end{array}$ & Talu & Mimosaceae & Herb & $\begin{array}{l}\text { Young leaves } \\
\text { and shoots are } \\
\text { used as vegetable }\end{array}$ \\
\hline
\end{tabular}


Table 2. Continued.

\begin{tabular}{|c|c|c|c|c|c|}
\hline S. No. & Botanical name & Local name & Family & Habit & Uses \\
\hline 66 & $\begin{array}{ll}\text { Parkia } & \text { roxburghii } \\
\text { G.Don. } & \end{array}$ & $\begin{array}{l}\text { Tupum Ash } \\
\text { Schein }\end{array}$ & Mimosaceae & Tree & $\begin{array}{l}\text { Young fruits } \\
\text { used as vegetable }\end{array}$ \\
\hline 67 & $\begin{array}{lr}\begin{array}{l}\text { Agrimonia } \\
\text { nepalensis }\end{array} & \text { (D.Don) } \\
\text { Syn. } & \text { Agrimonia } \\
\text { nepalensis D.Don. }\end{array}$ & Tanion & Rosaceae & Herb & $\begin{array}{l}\text { Leaves are used } \\
\text { as vegetable }\end{array}$ \\
\hline 68 & $\begin{array}{l}\text { Duchenea indica } \\
\text { (Andr.) Focke. Syn. } \\
\text { Fragaria indica }\end{array}$ & Nenye pepror & Rosaceae & Herb & Fruits are edible \\
\hline 69 & Prunus persica Linn. & Chekom & Rosaceae & Tree & Fruits are edible \\
\hline 70 & Prunus domestica Linn. & $\begin{array}{l}\text { Naaspai } \\
\text { Schein }\end{array}$ & Rosaceae & Tree & Fruits are edible \\
\hline 71 & Pyrus pashia D.Don. & Kean Schein & Rosaceae & Tree & Fruits are edible \\
\hline 72 & Rubus ellipticus Smith. & $\begin{array}{l}\text { Ta Hinch, Pop } \\
\text { taro }\end{array}$ & Rosaceae & Shrub & Fruits are edible \\
\hline 73 & Rubus hexagnus Roxb. & Ta Hinch & Rosaceae & Shrub & $\begin{array}{l}\text { Leaves used as } \\
\text { masticators }\end{array}$ \\
\hline 74 & Rubus insignis Hk.f. & Pop taro & Rosaceae & Shrub & Fruits are edible \\
\hline 75 & moluccanus & Taa Hich & Rosaceae & Shrub & Fruits are edible \\
\hline 76 & $\begin{array}{l}\text { Hydrangea robusta } \\
\text { Hook.f. }\end{array}$ & Ao Hagmi & Hydrangeaceae & Shrub & $\begin{array}{l}\text { Leaves are used } \\
\text { as vegetable }\end{array}$ \\
\hline 77 & $\begin{array}{l}\text { Terminalia chebula } \\
\text { Retz. }\end{array}$ & Bunura & Combretaceae & Tree & Fruits are edible \\
\hline 78 & $\begin{array}{l}\text { Terminalia citrina } \\
\text { (Gaerther) Flim. }\end{array}$ & Hilika & Combretaceae & Tree & Fruits are edible \\
\hline 79 & Psidium guajava Linn. & Modhori & Myrtaceae & Tree & Fruits are edible \\
\hline 80 & $\begin{array}{l}\text { Syzgium cumini (L.) } \\
\text { Skeels. }\end{array}$ & Jamun Schein & Myrtaceae & Tree & Fruits are edible \\
\hline 81 & $\begin{array}{lr}\text { Syzgium } & \text { fruticosum } \\
\text { DC.Syn. } & \text { Eugenia } \\
\text { fruticosa } & \text { Roxb. }\end{array}$ & Kurak Schein & Myrtaceae & Tree & Fruits are edible \\
\hline 82 & $\begin{array}{l}\text { Melastoma } \\
\text { malabathrium Linn. }\end{array}$ & $\begin{array}{l}\text { Doy Oppu } \\
\text { Yuppa Bo }\end{array}$ & Melastomataceae & Shrub & Fruits are edible \\
\hline 83 & $\begin{array}{l}\text { Melastoma normale } \\
\text { D.don. }\end{array}$ & Sie-Dhasha & Melastomataceae & Shrub & $\begin{array}{l}\text { Flowers and } \\
\text { ripened fruits are } \\
\text { edible }\end{array}$ \\
\hline 84 & $\begin{array}{l}\text { Oxyspora cenua } \\
\text { (Roxb.) Hook.F.\& } \\
\text { Thomson ex triana }\end{array}$ & - & Melastomataceae & Shrub & $\begin{array}{l}\text { Bark is removed } \\
\text { from stem and } \\
\text { eaten raw }\end{array}$ \\
\hline 85 & $\begin{array}{l}\text { Oreocnide integrifolia } \\
\text { (Gaud.)Miq.syn. } \\
\text { Oxyspora paniculata } \\
\text { DC. }\end{array}$ & Pokeerjali & Melastomataceae & Shrub & $\begin{array}{l}\text { Stem is eaten } \\
\text { raw }\end{array}$ \\
\hline 86 & $\begin{array}{l}\text { Osbeckia } \\
\text { Hooker. }\end{array}$ & Pudrasa & Melastomataceae & Shrub & $\begin{array}{l}\text { Fruits } \\
\text { occasionally } \\
\text { eaten by children }\end{array}$ \\
\hline 87 & $\begin{array}{l}\text { Osbeckia nutans } \\
\text { Wallich ex Clarke. }\end{array}$ & Rasa & Melastomataceae & Shrub & $\begin{array}{l}\text { Fruits are liked } \\
\text { by children }\end{array}$ \\
\hline 88 & $\begin{array}{l}\text { Sonerila } \\
\text { Roxb. }\end{array}$ & Jakmalo & Melastomataceae & Herb & $\begin{array}{l}\text { Leaves are used } \\
\text { as vegetable }\end{array}$ \\
\hline 89 & $\begin{array}{l}\text { Sonerila } \\
\text { Roxb. }\end{array}$ & Takmolo & Melastomataceae & Herb & $\begin{array}{l}\text { Leaves are used } \\
\text { as vegetable }\end{array}$ \\
\hline
\end{tabular}


Table 2. Continued.

\begin{tabular}{|c|c|c|c|c|c|}
\hline S. No. & Botanical name & Local name & Family & Habit & Uses \\
\hline 90 & Punica granatum Linn. & Anar & Punicaceae & Shrub & Fruits are edible \\
\hline 91 & Carica papaya Linn. & Omita Schein & Caricaceae & Tree & $\begin{array}{l}\text { Unripe fruits and } \\
\text { flowers are used } \\
\text { as vegetable }\end{array}$ \\
\hline 92 & $\begin{array}{l}\text { Cucubita maxima } \\
\text { Duchesne in Lamk. }\end{array}$ & Tар Рауо & Cucurbitaceae & Shrub & $\begin{array}{l}\text { Fruits are used as } \\
\text { vegetable }\end{array}$ \\
\hline 93 & Cucumis melo Linn. & Meble & Cucurbitaceae & Shrub & Fruits are edible \\
\hline 94 & $\begin{array}{l}\text { Cucurbita moschata } \\
\text { Duchesne }\end{array}$ & Tap & Cucurbitaceae & Climber & $\begin{array}{l}\text { Fruits, young } \\
\text { leaf tendrils, } \\
\text { flowers used as } \\
\text { vegetable }\end{array}$ \\
\hline 96 & Cucumis sativis Linn. & Mukku & Cucurbitaceae & Shrub & Fruits are edible \\
\hline 97 & Cucurbita pepo DC. & Loah & Cucurbitaceae & Shrub & $\begin{array}{l}\text { Fruits and leaves } \\
\text { used as vegetable }\end{array}$ \\
\hline 98 & Lagenaria vulgaris Ser. & Opu, & Cucurbitaceae & Climber & $\begin{array}{l}\text { Tender leaves } \\
\text { are used as } \\
\text { vegetable }\end{array}$ \\
\hline 99 & $\begin{array}{l}\text { Luffa acutangula Linn. } \\
\text { Syn. L. acutangula var. } \\
\text { amara Roxb. }\end{array}$ & Jhika & Cucurbitaceae & Climber & $\begin{array}{l}\text { Fruits and young } \\
\text { leaves are used } \\
\text { as vegetable }\end{array}$ \\
\hline 100 & Luffa aegyptiaca Mill. & Bhol & Cucurbitaceae & Climber & $\begin{array}{l}\text { Fruits are used as } \\
\text { vegetable }\end{array}$ \\
\hline 101 & $\begin{array}{l}\text { Momordica } \\
\text { cochinchinensis (Lour.) } \\
\text { Sprieng. }\end{array}$ & Bhat Kerela & Cucurbitaceae & Climber & $\begin{array}{l}\text { Fruits are used as } \\
\text { vegetable }\end{array}$ \\
\hline 102 & $\begin{array}{l}\text { Momordica charantia } \\
\text { Linn. }\end{array}$ & $\begin{array}{l}\text { Khechak } \\
\text { Kerela }\end{array}$ & Cucurbitaceae & Climber & $\begin{array}{l}\text { Fruits are bitter } \\
\text { in taste, used as } \\
\text { vegetable }\end{array}$ \\
\hline 103 & $\begin{array}{l}\text { Trichosanthes anbuina } \\
\text { Linn. }\end{array}$ & Dhunduli & Cucurbitaceae & Climber & $\begin{array}{l}\text { Fruits are used as } \\
\text { vegetable }\end{array}$ \\
\hline 104 & $\begin{array}{l}\text { Trichosanthes dioca } \\
\text { Roxb. }\end{array}$ & Duli Ao & Cucurbitaceae & Climber & $\begin{array}{l}\text { Fruits and leaves } \\
\text { are used as } \\
\text { vegetable }\end{array}$ \\
\hline 105 & Opuntia dillenii How. & Tha & Cactaceae & Shrub & Fruits are edible \\
\hline 106 & $\begin{array}{l}\text { Begonia palmata } \\
\text { D.Don.syn. B. laciniata } \\
\text { Roxb. }\end{array}$ & Bikku yulu & Begoniaceae & Herb & $\begin{array}{l}\text { Raw stem of } \\
\text { plant is used as } \\
\text { vegetable }\end{array}$ \\
\hline 107 & $\begin{array}{l}\text { Begonia roxburghii } \\
\text { (Miq.) DC.Prodr. }\end{array}$ & Bikku yulu & Begoniaceae & Herb & $\begin{array}{l}\text { Raw stem used } \\
\text { as vegetable }\end{array}$ \\
\hline 108 & $\begin{array}{l}\text { Centella asiatica (L.) } \\
\text { Urb. Syn. Hodocotyle } \\
\text { asiatica L. }\end{array}$ & Nguri & Apiacaee & Herb & $\begin{array}{l}\text { Leaves are used } \\
\text { as vegetable }\end{array}$ \\
\hline 109 & $\begin{array}{l}\text { Coriandrum sativum } \\
\text { Linn. }\end{array}$ & Dhania & Apiacaee & Herb & $\begin{array}{l}\text { Fruits and leaves } \\
\text { are used as spice }\end{array}$ \\
\hline 110 & $\begin{array}{l}\text { Erygnium foetidum } \\
\text { Linn. }\end{array}$ & Dhaniya pat & Apiacaee & Herb & $\begin{array}{l}\text { Leaves are used } \\
\text { for flavor curry }\end{array}$ \\
\hline 111 & $\begin{array}{l}\text { Oenanthe javanica } \\
\text { (Blume) DC. Syn. O. } \\
\text { benghalensis (DC.) } \\
\text { Benth. \& Hook.f. }\end{array}$ & Aguhama Ao & Apiacaee & Herb & $\begin{array}{l}\text { Whole plant is } \\
\text { used as vegetable }\end{array}$ \\
\hline
\end{tabular}


Table 2. Continued.

\begin{tabular}{|c|c|c|c|c|c|}
\hline S. No. & Botanical name & Local name & Family & Habit & Uses \\
\hline 112 & $\begin{array}{l}\text { Aralia arnata (G.Don) } \\
\text { Seen }\end{array}$ & Thang Ao & Araliaceae & Tree & $\begin{array}{l}\text { Leaves are used } \\
\text { as vegetable }\end{array}$ \\
\hline 113 & $\begin{array}{l}\text { Brassiopsis } \\
\text { glomerulata (Bl.) } \\
\text { Regel. Gaertn. Syn. B. } \\
\text { speciosa Deene and } \\
\text { Planch }\end{array}$ & Tago & Araliaceae & Tree & Fruits are edible \\
\hline 114 & $\begin{array}{l}\text { Mussaenda roxburghii } \\
\text { Hook. f. }\end{array}$ & Pattaa Tochar & Rubiaceae & Shrub & $\begin{array}{lr}\text { Young } & \text { leaves } \\
\text { and } & \text { flowers } \\
\text { eaten by } & \text { Tagin } \\
\text { tribe } & \end{array}$ \\
\hline 115 & $\begin{array}{l}\text { Mycetia longifolia } \\
\text { (Wall.) O. Klze. Syn. } \\
\text { Adenosacme longifolia } \\
\text { Wallich. }\end{array}$ & Tagmge & Rubiaceae & Shrub & $\begin{array}{l}\text { Leaves cooked } \\
\text { as vegetable }\end{array}$ \\
\hline 116 & Paedaria foetida Linn. & Upter Nemi & Rubiaceae & Climber & $\begin{array}{l}\text { Whole plant used } \\
\text { as vegetable }\end{array}$ \\
\hline 117 & $\begin{array}{l}\text { Spiradiclis bifida } \\
\text { Wallich ex Kurz. }\end{array}$ & Sokko & Rubiaceae & Herb & $\begin{array}{l}\text { Boiled leaves } \\
\text { used as vegetable }\end{array}$ \\
\hline 118 & $\begin{array}{l}\text { Bidens biternata (Lour) } \\
\text { Merr. \& Scherff ex } \\
\text { Scherff.FT }\end{array}$ & $\begin{array}{l}\text { Tagaem } \\
\text { Nyenyam }\end{array}$ & Asteraceae & Herb & $\begin{array}{l}\text { Young tender } \\
\text { leaves are eaten }\end{array}$ \\
\hline 119 & $\begin{array}{l}\text { Blumea fistulosa } \\
\text { (Roxb.) Kurz. }\end{array}$ & Rumdum & Asteraceae & Herb & $\begin{array}{l}\text { Whole plant used } \\
\text { as vegetable }\end{array}$ \\
\hline 120 & $\begin{array}{l}\text { Blumea lanceolariia } \\
\text { (Roxb.) Druce. Syn. B. } \\
\text { myriocephala DC. }\end{array}$ & - & Asteraceae & Shrub & $\begin{array}{l}\text { Leaves are used } \\
\text { as vegetable }\end{array}$ \\
\hline 121 & $\begin{array}{l}\text { Crassocephalum } \\
\text { crepidiodes (Benth) } \\
\text { Syn. } \quad \text { Gynura } \\
\text { crepidiodes Benth. }\end{array}$ & $\begin{array}{l}\text { Yamen, } \\
\text { Pakcho }\end{array}$ & Asteraceae & Herb & $\begin{array}{l}\text { Leaves are used } \\
\text { as vegetable }\end{array}$ \\
\hline 122 & $\begin{array}{l}\text { Dichrocephala latifolia } \\
\text { DC. }\end{array}$ & Pechikai & Asteraceae & Herb & $\begin{array}{l}\text { Tender leaves } \\
\text { are used as } \\
\text { vegetable }\end{array}$ \\
\hline 123 & Emilia sonchifolia DC. & Genta Ao & Asteraceae & Herb & $\begin{array}{l}\text { Leaves are used } \\
\text { as vegetable }\end{array}$ \\
\hline 124 & $\begin{array}{l}\text { Galinsoga parviflora } \\
\text { Cav. }\end{array}$ & Tedar Ao & Asteraceae & Herb & $\begin{array}{l}\text { Whole plant is } \\
\text { used as vegetable }\end{array}$ \\
\hline 125 & Sonchus arvensis Linn. & Tok Ruru Ao & Asteraceae & Herb & $\begin{array}{l}\text { Whole plant is } \\
\text { used as vegetable }\end{array}$ \\
\hline 126 & Spilanthes oleoracea & Motum Mersh & Asteraceae & Shrub & $\begin{array}{l}\text { Leaves and } \\
\text { inflorescence are } \\
\text { used as vegetable }\end{array}$ \\
\hline 127 & $\begin{array}{l}\text { Spilanthes paniculata } \\
\text { DC. }\end{array}$ & Mersha Ao & Asteraceae & Herb & $\begin{array}{l}\text { Young leaves are } \\
\text { used as vegetable }\end{array}$ \\
\hline 128 & $\begin{array}{lr}\text { Ardisia } & \text { thyrsiflora } \\
\text { D.Don.Syn. } & \text { Ardisia } \\
\text { nerifolia DC. } & \end{array}$ & Tujum Aep & Myrsinaceae & Tree & Fruits are edible \\
\hline 129 & $\begin{array}{l}\text { Argtreia nervosa } \\
\text { (Burm.f.) Bojer. Syn. A. } \\
\text { speciosa }\end{array}$ & - & Convolvulaceae & Climber & $\begin{array}{l}\text { Young shoots are } \\
\text { used as vegetable }\end{array}$ \\
\hline
\end{tabular}


Table 2. Continued.

\begin{tabular}{|c|c|c|c|c|c|}
\hline S. No. & Botanical name & Local name & Family & Habit & Uses \\
\hline 130 & $\begin{array}{l}\text { Ipomea batatas } \quad \text { L. } \\
\text { (Lamk.) }\end{array}$ & Eghein Phegre & Convolvulaceae & Climber & $\begin{array}{l}\text { Young tendrils } \\
\text { with leaves used } \\
\text { as vegetable. } \\
\text { Baked tuber also } \\
\text { eaten }\end{array}$ \\
\hline 131 & Capsicum annum Linn. & Yuluk, Yaluk & Solanaceae & Herb & $\begin{array}{l}\text { Fruits are used as } \\
\text { vegetable }\end{array}$ \\
\hline 132 & $\begin{array}{l}\text { Lycopersium } \\
\text { esculentum Miller. }\end{array}$ & Tomator & Solanaceae & Herb & $\begin{array}{l}\text { Fruits are used as } \\
\text { vegetable }\end{array}$ \\
\hline 133 & $\begin{array}{l}\text { Solanum melongena } \\
\text { Linn. }\end{array}$ & Bayom & Solanaceae & Herb & $\begin{array}{l}\text { Fruits are used as } \\
\text { vegetable }\end{array}$ \\
\hline 134 & Solanum nigrum Linn. & Hor & Solanaceae & Herb & $\begin{array}{l}\text { Fruits are eaten } \\
\text { when ripened }\end{array}$ \\
\hline 135 & $\begin{array}{l}\text { Solanum torvum } \\
\text { Swartz. }\end{array}$ & Shoat Beyak & Solanaceae & Shrub & Fruits are edible \\
\hline 136 & $\begin{array}{l}\text { Solanum tuberosum } \\
\text { Linn. }\end{array}$ & Alu & Solanaceae & Herb & $\begin{array}{l}\text { Tuber is used as } \\
\text { vegetable }\end{array}$ \\
\hline 137 & Physalis peruviana L. & Donam As & Solanaceae & Herb & Fruits are edible \\
\hline 138 & $\begin{array}{ll}\text { Boeica } & \text { filiformis } \\
\text { C.B.Clarke } & \end{array}$ & Jookey & Gesneriaceae & Shrub & $\begin{array}{l}\text { Leaves are used } \\
\text { as vegetable }\end{array}$ \\
\hline 139 & $\begin{array}{ll}\text { Boeica } & \text { fulva } \\
\text { C.B.Clarke } & \end{array}$ & Joke Ao & Gesneriaceae & Herb & $\begin{array}{l}\text { Leaves are used } \\
\text { as vegetable }\end{array}$ \\
\hline 140 & $\begin{array}{l}\text { Rhynchotechum } \\
\text { ellipticum (Wallich ex } \\
\text { Dietr.) A.DC. }\end{array}$ & Jooke & Gesneriaceae & Shrub & $\begin{array}{l}\text { Leaves are used } \\
\text { as vegetable }\end{array}$ \\
\hline 141 & $\begin{array}{l}\text { Phlogacanthus } \\
\text { thyrsiflorous Nees. }\end{array}$ & Pilamola & Acanthaceae & Shrub & $\begin{array}{l}\text { Red flowers are } \\
\text { used as vegetable }\end{array}$ \\
\hline 142 & $\begin{array}{l}\text { Strobilanthus furcatus } \\
\text { Biswas }\end{array}$ & Barche Ao & Acanthaceae & Shrub & $\begin{array}{l}\text { Leaves and } \\
\text { young shoots are } \\
\text { edible }\end{array}$ \\
\hline 143 & $\begin{array}{l}\text { Callicarpa macrophylla } \\
\text { Vahl. }\end{array}$ & Upneu & Verbenaceae & Shrub & Fruits are edible \\
\hline 144 & $\begin{array}{l}\text { Callicarpa vestila Wall. } \\
\text { ex } \mathrm{Cl}\end{array}$ & Yalu & Verbenaceae & Shrub & $\begin{array}{l}\text { Dried stem is } \\
\text { used as substitute } \\
\text { for tobacco }\end{array}$ \\
\hline 145 & $\begin{array}{l}\text { Clerodendron } \\
\text { colebrookianum Walp. }\end{array}$ & Tippin & Verbenaceae & Shrub & $\begin{array}{l}\text { Leaves are used } \\
\text { as vegetable }\end{array}$ \\
\hline 146 & $\begin{array}{l}\text { Clerodendron serratum } \\
\text { (Linn.) Moon. }\end{array}$ & Tipin poto & Verbenaceae & Shrub & $\begin{array}{l}\text { Leaves are used } \\
\text { as vegetable }\end{array}$ \\
\hline 147 & $\begin{array}{l}\text { Clerodendron viscosum } \\
\text { Vent. Syn. C. C. } \\
\text { infortunatum Gaertn. }\end{array}$ & Taapin & Verbenaceae & Shrub & $\begin{array}{l}\text { Flowers are used } \\
\text { as vegetable }\end{array}$ \\
\hline 148 & Gmelina arborea Roxb. & Gomori Schein & Verbenaceae & Tree & $\begin{array}{l}\text { Flowers are used } \\
\text { as vegetable }\end{array}$ \\
\hline 149 & $\begin{array}{l}\text { Premma milleflora } \\
\text { C.B.Clarke. }\end{array}$ & - & Verbenaceae & Tree & $\begin{array}{l}\text { Fruits and leaves } \\
\text { are edible }\end{array}$ \\
\hline 150 & $\begin{array}{l}\text { Ajuga macrosperma } \\
\text { Wall. }\end{array}$ & Nomdemghor & Lamiaceae & Herb & $\begin{array}{l}\text { Whole plant is } \\
\text { used as vegetable }\end{array}$ \\
\hline 151 & $\begin{array}{l}\text { Mentha piperata Linn. } \\
\text { Emend. Huds. }\end{array}$ & Pudina & Lamiaceae & Herb & $\begin{array}{l}\text { Leaves are used } \\
\text { in salad and } \\
\text { making chutni }\end{array}$ \\
\hline
\end{tabular}


Table 2. Continued.

\begin{tabular}{|c|c|c|c|c|c|}
\hline S. No. & Botanical name & Local name & Family & Habit & Uses \\
\hline 152 & $\begin{array}{l}\text { Perilla frustescens (L.) } \\
\text { Britt. }\end{array}$ & Tanam & Lamiaceae & Shrub & $\begin{array}{l}\text { Seeds are used as } \\
\text { spice ingredient }\end{array}$ \\
\hline 153 & $\begin{array}{l}\text { Plantago erosa Wall. } \\
\text { Syn. P. major Linn. }\end{array}$ & Talak Ao & Plantaginaceae & Herb & $\begin{array}{l}\text { Leaves are used } \\
\text { as vegetable }\end{array}$ \\
\hline 154 & $\begin{array}{l}\text { Amaranthus gangeticus } \\
\text { Linn. }\end{array}$ & Data Ao & Amaranthaceae & Herb & $\begin{array}{l}\text { Whole plant is } \\
\text { used as vegetable }\end{array}$ \\
\hline 155 & $\begin{array}{l}\text { Amaranthus spinosus } \\
\text { Linn. }\end{array}$ & Puchu Panya & Amaranthaceae & Herb & $\begin{array}{l}\text { Leaves are used } \\
\text { as vegetable }\end{array}$ \\
\hline 156 & $\begin{array}{l}\text { Amaranthus viridis } \\
\text { Linn. }\end{array}$ & Detta Ao & Amaranthaceae & Herb & $\begin{array}{l}\text { Leaves are used } \\
\text { as vegetable }\end{array}$ \\
\hline 157 & Celosia argentea Linn. & - & Amaranthaceae & Herb & $\begin{array}{l}\text { Leaves are used } \\
\text { as vegetable }\end{array}$ \\
\hline 158 & $\begin{array}{l}\text { Chenopodium album } \\
\text { Linn. }\end{array}$ & Teya Ao & Chenopodaceae & Herb & $\begin{array}{l}\text { Leaves are used } \\
\text { as vegetable }\end{array}$ \\
\hline 159 & $\begin{array}{l}\text { Chenopodium } \\
\text { ambrosioides Linn. }\end{array}$ & Teya & Chenopodaceae & Herb & $\begin{array}{l}\text { Leaves are used } \\
\text { as vegetable }\end{array}$ \\
\hline 160 & $\begin{array}{l}\text { Fagopyrum dibotrys } \\
\text { (D.Don.) Trev. Syn. F. } \\
\text { cymosum }\end{array}$ & Hukku & Polygonaceae & Herb & $\begin{array}{l}\text { Leaves are used } \\
\text { as vegetable }\end{array}$ \\
\hline 161 & $\begin{array}{l}\text { Fagopyrum esculentum } \\
\text { Moench. }\end{array}$ & $\begin{array}{l}\text { Hukku, } \\
\text { Hukkung }\end{array}$ & Polygonaceae & Herb & $\begin{array}{l}\text { Leaves are used } \\
\text { as vegetable }\end{array}$ \\
\hline 162 & 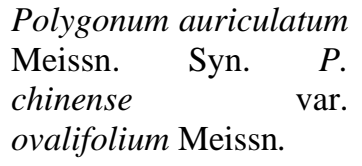 & Torckumbuk & Polygonaceae & Herb & Fruits are edible \\
\hline 163 & $\begin{array}{l}\text { Polygonum posumbu } \\
\text { Ham. }\end{array}$ & Namne Ao & Polygonaceae & Climber & $\begin{array}{l}\text { Leaves are used } \\
\text { as vegetable }\end{array}$ \\
\hline 164 & $\begin{array}{l}\text { Polygonum runcinatum } \\
\text { Ham. }\end{array}$ & Puri & Polygonaceae & Herb & $\begin{array}{l}\text { Leaves are used } \\
\text { as vegetable }\end{array}$ \\
\hline 165 & $\begin{array}{l}\text { Rumex } \quad \text { nepalensis } \\
\text { Spring }\end{array}$ & Yalak Ao & Polygonaceae & Herb & $\begin{array}{l}\text { Leaves are used } \\
\text { as vegetable }\end{array}$ \\
\hline 166 & Piper betle Linn. & Pan & Piperaceae & Climber & $\begin{array}{l}\text { Leaves are eaten } \\
\text { with Areca nut }\end{array}$ \\
\hline 167 & Piper nigrum Linn. & Jaluk & Piperaceae & Shrub & $\begin{array}{l}\text { Fruits are used as } \\
\text { spice }\end{array}$ \\
\hline 168 & $\begin{array}{l}\text { Piper pedicellosum } \\
\text { Wall. }\end{array}$ & Rer & Piperaceae & Shrub & $\begin{array}{l}\text { Leaves are used } \\
\text { as vegetable }\end{array}$ \\
\hline 169 & $\begin{array}{l}\text { Houttunyia cordata } \\
\text { Thumb. }\end{array}$ & Checha Peya & Saururaceae & Herb & $\begin{array}{l}\text { Raw leaves are } \\
\text { used as vegetable } \\
\text { and chatni }\end{array}$ \\
\hline 170 & $\begin{array}{l}\text { Chloranthus glaber } \\
\text { (Thumb.) Makino Syn. } \\
\text { C. brachystachya } \\
\text { Blume. }\end{array}$ & Tupu Tal & Chloranthaceae & Shrub & Fruits are edible \\
\hline 171 & $\begin{array}{l}\text { Cinnamomum tamala } \\
\text { (Buch-Ham.) Nees \& } \\
\text { Ebern. }\end{array}$ & Tesh patta & Lauraceae & Tree & $\begin{array}{l}\text { Leaves are used } \\
\text { as spice }\end{array}$ \\
\hline 172 & $\begin{array}{l}\text { Cinnamomum } \\
\text { zeylanicum Breya. }\end{array}$ & Dalchini & Lauraceae & Tree & $\begin{array}{l}\text { Bark is used as } \\
\text { spice }\end{array}$ \\
\hline 173 & $\begin{array}{l}\text { Litsea cubeba (Lour) } \\
\text { Pers. Syn. L. citrata Bl. }\end{array}$ & $\begin{array}{ll}\text { Teer, } & \text { Tayir } \\
\text { Schein } & \\
\end{array}$ & Lauraceae & Tree & Fruits are edible \\
\hline
\end{tabular}


Table 2. Continued.

\begin{tabular}{|c|c|c|c|c|c|}
\hline S. No. & Botanical name & Local name & Family & Habit & Uses \\
\hline 174 & $\begin{array}{l}\text { Litsea salicifolia (Roxb. } \\
\text { ex Nees) Hk.f. }\end{array}$ & Taor & Lauraceae & Tree & Fruits are edible \\
\hline 175 & $\begin{array}{l}\text { Persea frutifera Kost. } \\
\text { Syn. Machilus edulis } \\
\text { King ex Hk.f. }\end{array}$ & Techir Shein & Lauraceae & Tree & Fruits are edible \\
\hline 176 & $\begin{array}{l}\text { Phoebe lanceolata } \\
\text { (Nees) Nees. }\end{array}$ & Sichir Schein & Lauraceae & Tree & Fruits are edible \\
\hline 177 & $\begin{array}{l}\text { Elaeagnus latifolia } \\
\text { Linn. }\end{array}$ & Hari & Elaegnaceae & Shrub & $\begin{array}{l}\text { Fruits with sour } \\
\text { taste are eaten }\end{array}$ \\
\hline 178 & $\begin{array}{l}\text { Baccaaurea sapida } \\
\text { (Roxb.) Muell. }\end{array}$ & Bheri Aash & Euphorbiaceae & Tree & Fruits are edible \\
\hline 179 & $\begin{array}{l}\text { Baliosperum calycinum } \\
\text { Muell-Arg. }\end{array}$ & - & Euphorbiaceae & Tree & $\begin{array}{lr}\text { Leaves } & \text { are } \\
\text { cooked } & \text { as } \\
\text { vegetable } & \end{array}$ \\
\hline 180 & $\begin{array}{l}\text { Baliosperum montanum } \\
\text { (Willd.) Muell.Arg. } \\
\text { Syn. B. axillana }\end{array}$ & Pririya & Euphorbiaceae & Shrub & $\begin{array}{l}\text { Leaves are used } \\
\text { as vegetable }\end{array}$ \\
\hline 181 & Euphorbia hirta Linn. & Tamu Ao & Euphorbiaceae & Herb & $\begin{array}{l}\text { Leaves are used } \\
\text { as vegetable }\end{array}$ \\
\hline 182 & roxburghii & Tuy Tya & Euphorbiaceae & Shrub & Fruits are edible \\
\hline 183 & $\begin{array}{l}\text { Manihot esculenta } \\
\text { Crantz. }\end{array}$ & Sida Eighein & Euphorbiaceae & Shrub & $\begin{array}{l}\text { Leaves and } \\
\text { tubers are used } \\
\text { as vegetable }\end{array}$ \\
\hline 184 & $\begin{array}{l}\text { Phyllanthus emblica L. } \\
\text { Hk. f. }\end{array}$ & Amlaki Schein & Euphorbiaceae & Tree & Fruits are edible \\
\hline 185 & $\begin{array}{l}\text { Elatostema } \\
\text { platyphyllum Wedd. }\end{array}$ & Hoj Ao & Urticaceae & Shrub & $\begin{array}{l}\text { Leaves are used } \\
\text { as vegetable }\end{array}$ \\
\hline 186 & $\begin{array}{l}\text { Elatostema sessile } \\
\text { Frost. }\end{array}$ & Jooke & Urticaceae & Herb & $\begin{array}{l}\text { Leaves are used } \\
\text { as vegetable }\end{array}$ \\
\hline 187 & $\begin{array}{l}\text { Laportea crenulata } \\
\text { Gaud. }\end{array}$ & $\begin{array}{l}\text { Pud } \\
\text { Raate }\end{array}$ & Urticaceae & Shrub & $\begin{array}{l}\text { Young leaves } \\
\text { used as vegetable }\end{array}$ \\
\hline 188 & Pilea bracteosa Wedd. & Guge & Urticaceae & Herb & $\begin{array}{l}\text { Leaves used as } \\
\text { vegetable }\end{array}$ \\
\hline 189 & $\begin{array}{l}\text { Pilea glaberrima Bl. } \\
\text { Syn. P. } \quad \text { smilacifolia } \\
\text { Wedd. }\end{array}$ & Guge Ao & Urticaceae & Herb & $\begin{array}{l}\text { Leaves are used } \\
\text { as vegetable }\end{array}$ \\
\hline 190 & $\begin{array}{l}\text { Pouzolzia bennetiana } \\
\text { Wight. }\end{array}$ & Huyiek & Urticaceae & Climber & $\begin{array}{l}\text { Leaves are used } \\
\text { as vegetable }\end{array}$ \\
\hline 191 & $\begin{array}{l}\text { Pouzolzia } \\
\text { (Bl.)Merr. }\end{array}$ & Tanu & Urticaceae & Shrub & $\begin{array}{l}\text { Leaves are used } \\
\text { as vegetable. } \\
\text { Tubers used as } \\
\text { pig feed }\end{array}$ \\
\hline 192 & $\begin{array}{l}\text { Pouzolzia sanguine } \\
\text { (Bl.) Merr.var. fulgens }\end{array}$ & Tanu Nenya & Urticaceae & Tree & $\begin{array}{l}\text { Leaves are used } \\
\text { as vegetable. }\end{array}$ \\
\hline & $\begin{array}{l}\text { (Wedd.) Syn. } \quad P . \\
\text { viminea Wedd. } \\
\text { fulgens Wedd. }\end{array}$ & & & & $\begin{array}{l}\text { Tubers are used } \\
\text { as pig feed }\end{array}$ \\
\hline 193 & $\begin{array}{l}\text { Bischofia javanica } \\
\text { Blume. }\end{array}$ & Tag Schein & Bischofiaceae & Tree & $\begin{array}{l}\text { Young leaves are } \\
\text { used as vegetable }\end{array}$ \\
\hline
\end{tabular}


Table 2. Continued.

\begin{tabular}{|c|c|c|c|c|c|}
\hline S. No. & Botanical name & Local name & Family & Habit & Uses \\
\hline 194 & Artocarpus chama & Tak Sam Shein & Moraceae & Tree & Fruits are edible \\
\hline & $\begin{array}{l}\text { Buch. Syn. } \\
\text { chaplasha Roxb. }\end{array}$ & & & & \\
\hline 195 & $\begin{array}{l}\text { Artocarpus } \\
\text { heterophyllus } \quad \text { Lam. }\end{array}$ & Tak Bela & Moraceae & Tree & Fruits are edible \\
\hline & $\begin{array}{l}\text { Syn. A. integrifolius } \\
\text { non L. }\end{array}$ & & & & \\
\hline 196 & $\begin{array}{l}\text { Artocarpus lakoocha } \\
\text { Roxb. }\end{array}$ & $\begin{array}{l}\text { Bela Ninye } \\
\text { Schein }\end{array}$ & Moraceae & Tree & $\begin{array}{l}\text { Fruits are edible. } \\
\text { Bark is chewed } \\
\text { with pan (Betel) }\end{array}$ \\
\hline 197 & $\begin{array}{l}\text { Conocephalus } \\
\text { suaveolens non Blume }\end{array}$ & Hogen Ao & Moraceae & Climber & $\begin{array}{l}\text { Young tender } \\
\text { leaves used as } \\
\text { vegetable }\end{array}$ \\
\hline 198 & $\begin{array}{l}\text { Ficus auriculata Lour. } \\
\text { Syn. F. roxburghii } \\
\text { Wall. }\end{array}$ & Takuk & Moraceae & Tree & Fruits are edible \\
\hline 199 & Ficus elastica Roxb. & Sherak, Sangri & Moraceae & Tree & Fruits are edible \\
\hline 200 & Ficus fistulosa Reinw. & Mobopu & Moraceae & Shrub & Fruits are edible \\
\hline 201 & Ficus squamosa Roxb. & Talagi & Moraceae & Tree & Fruits are edible \\
\hline & $\begin{array}{l}\text { Syn. F. saemocarpa } \\
\text { Miq. }\end{array}$ & & & & \\
\hline 202 & Morus alba Linn. & Latek Schein & Moraceae & Tree & Fruits are edible \\
\hline 203 & Morus indica Linn. & Latek & Moraceae & Tree & Fruits are edible \\
\hline 204 & Juglans regia Linn. & Akrod & Juglanadaceae & Tree & Fruits are edible \\
\hline 205 & $\begin{array}{l}\text { Castanopsis indica } \\
\text { (Roxb.) A. DC. }\end{array}$ & Hinguri & Fagaceae & Tree & Fruits are edible \\
\hline 206 & $\begin{array}{lccc}\text { Castanopsis } & \text { lancifolia } \\
\text { (Roxb.) } & \text { Hockel } & \& & \text { A. } \\
\text { Camus. } & \text { Syn. } & & \text { C. } \\
\text { lancifolia } & & & \end{array}$ & Tain & Fagaceae & Tree & Fruits are edible \\
\hline 207 & $\begin{array}{l}\text { Castanopsis tribuloides } \\
\text { (Smith) A. DC. }\end{array}$ & Tain Tha & Fagaceae & Tree & Fruits are edible \\
\hline 208 & $\begin{array}{l}\text { Quercus griffithii Hook } \\
\text { f. \& Thoms. }\end{array}$ & Kra & Fagaceae & Tree & Fruits are edible \\
\hline 209 & $\begin{array}{l}\text { Quercus lamellose } \\
\text { Smith. }\end{array}$ & Khora Niddum & Fagaceae & Tree & Fruits are edible \\
\hline 210 & $\begin{array}{l}\text { Alpinia nigra (Gaertn.) } \\
\text { Burtt. Syn. A. allughas } \\
\text { (Retz.) Rosc. }\end{array}$ & Doyak & Zingiberaceae & Herb & Fruits are edible \\
\hline 211 & $\begin{array}{l}\text { Amomum aromaticum } \\
\text { Roxb. }\end{array}$ & Khobu Telli & Zingiberaceae & Herb & $\begin{array}{l}\text { Seeds are used as } \\
\text { spice }\end{array}$ \\
\hline 212 & $\begin{array}{l}\text { Amomum subulatam } \\
\text { Roxb. }\end{array}$ & $\begin{array}{l}\text { Mutum Khobu } \\
\text { Telli }\end{array}$ & Zingiberaceae & Herb & $\begin{array}{l}\text { Seeds are used as } \\
\text { spice }\end{array}$ \\
\hline 213 & Curcuma longa Linn. & Kaya Haldi & Zingiberaceae & Herb & $\begin{array}{l}\text { Rhizomes are } \\
\text { used as spice }\end{array}$ \\
\hline 214 & $\begin{array}{l}\text { Hedychium coccineum } \\
\text { J.E. Smith. }\end{array}$ & Aemmi Pekchi & Zingiberaceae & Herb & $\begin{array}{l}\text { Young tender } \\
\text { stem is used as } \\
\text { vegetable }\end{array}$ \\
\hline
\end{tabular}


Table 2. Continued.

\begin{tabular}{|c|c|c|c|c|c|}
\hline S. No. & Botanical name & Local name & Family & Habit & Uses \\
\hline 215 & $\begin{array}{l}\text { Hedychium spicatum } \\
\text { Lodd. }\end{array}$ & Telli & Zingiberaceae & Herb & Fruits are edible \\
\hline 216 & $\begin{array}{l}\text { Zingiber officinale } \\
\text { Rose. }\end{array}$ & Take & Zingiberaceae & Herb & $\begin{array}{l}\text { Rhizomes are } \\
\text { used as spice }\end{array}$ \\
\hline 217 & $\begin{array}{l}\text { Phrynium capitatum } \\
\text { Willd. }\end{array}$ & Khokam & Marantaceae & Shrub & $\begin{array}{l}\text { Leaves used as } \\
\text { packing material } \\
\text { for preparation } \\
\text { process of } \\
\text { traditional drinks }\end{array}$ \\
\hline 218 & $\begin{array}{l}\text { Phrynium imbricatum } \\
\text { Roxb. }\end{array}$ & $\begin{array}{l}\text { Kokam } \\
\text { Kumpu }\end{array}$ & Marantaceae & Shrub & As above \\
\hline 219 & $\begin{array}{l}\text { Ensete glaucum (Roxb.) } \\
\text { Syn. Musa glauca } \\
\text { Roxb. }\end{array}$ & Kudum & Musaceae & $\begin{array}{l}\text { Small } \\
\text { tree }\end{array}$ & Fruits are edible \\
\hline 220 & $\begin{array}{l}\text { Musa acuminata Colla. } \\
\text { Syn. } \quad \text { M. } \quad \text { sanguinea } \\
\text { Hook .f. }\end{array}$ & Kulu & Musaceae & $\begin{array}{l}\text { Small } \\
\text { tree }\end{array}$ & Fruits are edible \\
\hline 221 & $\begin{array}{l}\text { Musa bulbisiana Colla. } \\
\text { Syn. M. sapientum auct } \\
\text { non L. }\end{array}$ & Кора & Musaceae & $\begin{array}{l}\text { Small } \\
\text { tree }\end{array}$ & $\begin{array}{l}\text { Fruits and inner } \\
\text { soft stem are } \\
\text { edible }\end{array}$ \\
\hline 222 & $\begin{array}{l}\text { Musa paradisiacal } \\
\text { Linn. }\end{array}$ & $\begin{array}{l}\text { Nyepak Ao- } \\
\text { Kopak }\end{array}$ & Musaceae & $\begin{array}{l}\text { Small } \\
\text { Tree }\end{array}$ & $\begin{array}{l}\text { Unripe fruits } \\
\text { used as vegetable }\end{array}$ \\
\hline 223 & Musa velutina Wendl. & $\begin{array}{ll}\text { Anye } & \text { Kodok } \\
\text { Kolok } & \end{array}$ & Musaceae & $\begin{array}{l}\text { Small } \\
\text { Tree }\end{array}$ & $\begin{array}{l}\text { Roots and seeds } \\
\text { are edible }\end{array}$ \\
\hline 224 & $\begin{array}{lr}\text { Molineria } & \text { recurvata } \\
\text { Dryland. } & \text { Syn. } \\
\text { Curculigo } & \text { recurvata } \\
\text { Dryland. } & \end{array}$ & Doyak & Hypoxidaceae & Herb & Fruits are edible \\
\hline 225 & Dioscorea alata Linn. & $\begin{array}{l}\text { Eghen } \\
\text { Ngencha }\end{array}$ & Dioscoreaceae & Climber & Tubers are edible \\
\hline 226 & $\begin{array}{l}\text { Dioscorea belophylla } \\
\text { (Prain) Voigt }\end{array}$ & Eghen Tabon & Dioscoreaceae & Climber & Tubers are edible \\
\hline 227 & $\begin{array}{l}\text { Dioscorea bulbifera } \\
\text { Linn. }\end{array}$ & Helak & Dioscoreaceae & Climber & Tubers are edible \\
\hline 228 & $\begin{array}{l}\text { Dioscorea deltoidea } \\
\text { Wall ex. Griseb }\end{array}$ & Eghen Ngenki & Dioscoreaceae & Climber & Tubers are edible \\
\hline 229 & $\begin{array}{l}\text { Dioscorea glabra } \\
\text { Roxb. }\end{array}$ & $\begin{array}{l}\text { Eghen } \\
\text { Ngentak }\end{array}$ & Dioscoreaceae & Climber & Tubers are edible \\
\hline 230 & $\begin{array}{l}\text { Dioscorea hamiltonii } \\
\text { Hook. f. }\end{array}$ & Eghen Raad & Dioscoreaceae & Climber & Tubers are edible \\
\hline 231 & $\begin{array}{l}\text { Dioscorea laurifolia } \\
\text { Wall }\end{array}$ & Eghen Ngegek & Dioscoreaceae & Climber & Tubers are edible \\
\hline 232 & $\begin{array}{l}\text { Dioscorea } \\
\text { melanophylla }\end{array}$ & Hash & Dioscoreaceae & Climber & Tubers are edible \\
\hline 233 & $\begin{array}{l}\text { Dioscorea oppositifolia } \\
\text { Linn. }\end{array}$ & $\begin{array}{l}\text { Eghen Take- } \\
\text { Mare }\end{array}$ & Dioscoreaceae & Climber & Tubers are edible \\
\hline 234 & $\begin{array}{l}\text { Dioscorea orbiculata } \\
\text { Hook }\end{array}$ & Eghen Yeb & Dioscoreaceae & Climber & Tubers are edible \\
\hline 235 & $\begin{array}{l}\text { Dioscorea pentaphylla } \\
\text { Linn. }\end{array}$ & Hill & Dioscoreaceae & Climber & Tubers are edible \\
\hline
\end{tabular}


Table 2. Continued.

\begin{tabular}{|c|c|c|c|c|c|}
\hline S. No. & Botanical name & Local name & Family & Habit & Uses \\
\hline 236 & $\begin{array}{l}\text { Dioscorea purpurea } \\
\text { Roxb. }\end{array}$ & $\begin{array}{l}\text { Eghen } \\
\text { Ngenchu } \\
\text { Puyur }\end{array}$ & Dioscoreaceae & Climber & Tubers are edible \\
\hline 237 & $\begin{array}{l}\text { Dioscorea } \\
\text { Kunth }\end{array}$ & Eghen Ngenro & Dioscoreaceae & Climber & Tubers are edible \\
\hline 238 & Dioscorea spicata Roth & $\begin{array}{l}\text { Eghen } \\
\text { Ngenchi }\end{array}$ & Dioscoreaceae & Climber & Tubers are edible \\
\hline 239 & $\begin{array}{l}\text { Dioscorea wallichii } \\
\text { Hook. f. }\end{array}$ & Eghen Ngeyuk & Dioscoreaceae & Climber & Tubers are edible \\
\hline 240 & $\begin{array}{l}\text { Dioscorea wightii } \\
\text { Hook.f. }\end{array}$ & Eghen Ngebuk & Dioscoreaceae & Climber & Tubers are edible \\
\hline 241 & Allium cepa Linn. & Talap & Liliaceae & Herb & $\begin{array}{l}\text { Whole plant is } \\
\text { used as spice }\end{array}$ \\
\hline 242 & $\begin{array}{ll}\text { Allium } & \text { hookeri } \\
\text { Thwaites. } & \end{array}$ & Nyishi Talap & Liliaceae & Herb & $\begin{array}{l}\text { Bulb is used as } \\
\text { spice }\end{array}$ \\
\hline 243 & Allium sativum Linn. & Lohsum- & Liliaceae & Herb & $\begin{array}{l}\text { Bulb is used as } \\
\text { spice }\end{array}$ \\
\hline 244 & $\begin{array}{l}\text { Asparagus racemosus } \\
\text { Willd. }\end{array}$ & - & Liliaceae & Shrub & $\begin{array}{l}\text { Tuber and whole } \\
\text { plant has diuretic } \\
\text { and cooling } \\
\text { properties }\end{array}$ \\
\hline 245 & $\begin{array}{l}\text { Pollia hasskarlii Rao } \\
\text { Syn. P. acilisa Hassk. }\end{array}$ & Nipobapak & Commelinaceae & Herb & Fruits are edible \\
\hline 246 & Areca catechu Linn. & Tamol & Arecaceae & Tree & $\begin{array}{l}\text { Fruits are used as } \\
\text { masticator }\end{array}$ \\
\hline 247 & Calamus erectus Roxb. & Tare & Arecaceae & Shrub & $\begin{array}{l}\text { Young and soft } \\
\text { stem is edible }\end{array}$ \\
\hline 248 & $\begin{array}{l}\text { Calamus } \\
\text { Griff. }\end{array}$ & Tar Rame & Arecaceae & Cane & Fruits are edible \\
\hline 249 & $\begin{array}{l}\text { Calamus latifolius } \\
\text { Roxb. }\end{array}$ & Takat & Arecaceae & Cane & Fruits are edible \\
\hline 250 & Calamus tenuis Roxb. & Ter Remme & Arecaceae & Cane & Fruits are edible \\
\hline 251 & $\begin{array}{l}\text { Daemonorops } \\
\text { jeinkinsianus Griff. }\end{array}$ & Raidang & Arecaceae & Cane & Fruits are edible \\
\hline 252 & $\begin{array}{l}\text { Livistona jenkinsiana } \\
\text { Griff. }\end{array}$ & Taak & Arecaceae & Tree & $\begin{array}{l}\text { Fruits and tender } \\
\text { stem are edible }\end{array}$ \\
\hline 253 & $\begin{array}{l}\text { Pinnanga } \quad \text { gracilis } \\
\text { (Roxb.) Bl. }\end{array}$ & Taecher & Arecaceae & Tree & Fruits are edible \\
\hline 254 & $\begin{array}{l}\text { Wallichia densiflora } \\
\text { Mart. }\end{array}$ & Tache & Arecaceae & Tree & $\begin{array}{l}\text { Stem is used to } \\
\text { extract a flour } \\
\text { which is used as } \\
\text { famine food }\end{array}$ \\
\hline 255 & $\begin{array}{l}\text { Wallichia disticha } \mathrm{T} \text {. } \\
\text { Anders }\end{array}$ & Tali & Arecaceae & Tree & Fruits are eaten \\
\hline 256 & Zalacca secunda Griff & $\mathrm{Ra}$ & Are & Tree & Fruits are edible \\
\hline 257 & $\begin{array}{l}\text { Colocasia antiquorum } \\
\text { Schott Melet }\end{array}$ & Takche Reba & Araceae & Herb & $\begin{array}{l}\text { Tubers, stem and } \\
\text { leaves are used } \\
\text { as vegetable }\end{array}$ \\
\hline 258 & $\begin{array}{l}\text { Colocasia } \\
\text { Schott. }\end{array}$ & Nyepop & Araceae & Herb & As above \\
\hline 259 & Colocasia fallax Schott & Nygek & Araceae & Herb & As above \\
\hline
\end{tabular}


Table 2. Continued.

\begin{tabular}{|c|c|c|c|c|c|}
\hline S. No. & Botanical name & Local name & Family & Habit & Uses \\
\hline 260 & Colocasia esculenta & Nyepu yulu & Araceae & Herb & As above \\
\hline 261 & Cyperus rotundus Linn. & $\mathrm{Ei}$ & Cyperaceae & Herb & $\begin{array}{ll}\text { Roots are } & \text { by } \\
\text { chewed } & \text { byildren due } \\
\text { chile } & \\
\text { sweet taste } & \end{array}$ \\
\hline 262 & $\begin{array}{l}\text { Dendrocalamus } \\
\text { hamiltonii Nees et Arn. }\end{array}$ & $\mathrm{Ae}$ & Poaceae & Bamboo & $\begin{array}{l}\text { Young tender } \\
\text { shoots are eaten }\end{array}$ \\
\hline 263 & $\begin{array}{l}\text { Dendrocalamus } \\
\text { giganteus Munro }\end{array}$ & Ae Hatee & Poaceae & Bamboo & As above \\
\hline 264 & $\begin{array}{l}\text { Panicum milaceum } \\
\text { Linn. }\end{array}$ & Tai & Poaceae & Herb & Seeds are eaten \\
\hline 265 & $\begin{array}{l}\text { Saccharum spontaneum } \\
\text { Linn }\end{array}$ & Teppi & Poaceae & Shrub & $\begin{array}{l}\text { Young tender } \\
\text { stem is eaten }\end{array}$ \\
\hline 266 & Setaria italic Beav. & Tayak & Poaceae & Herb & $\begin{array}{l}\text { Leaves used for } \\
\text { making local } \\
\text { beverage }\end{array}$ \\
\hline 267 & Zea mays Linn & Tор & Poaceae & $\begin{array}{l}\text { Tall } \\
\text { herb }\end{array}$ & $\begin{array}{l}\text { Maize corns used } \\
\text { as staple food } \\
\text { and in local } \\
\text { beverage }\end{array}$ \\
\hline 268 & $\begin{array}{l}\text { Selaginella biformis Br. } \\
\text { ex Kuhn. }\end{array}$ & Husum Ao & Selaginelaceae & Herb & $\begin{array}{l}\text { Stem and leaves } \\
\text { are used as } \\
\text { vegetable }\end{array}$ \\
\hline 269 & Dillenia indica Linn & Jampa & Dillenaceae & Tree & Fruits are edible \\
\hline
\end{tabular}

From the ethno botanical survey, it was noted that tribes were consuming the edible plants in raw or cooked form. The plant parts used were fruits, leaves, tubers, whole plant, stem/bark, seeds and flowers in descending order (Figure $2)$. The fruits of 121 species are eaten as raw and sometimes made in to salads or pickles. Leaves of 84 species are used as vegetables.

Trees made the highest proportion of edible species followed by shrubs, herbs, climbers in descending order (Figure 3). The time and frequency of collecting various plants varied from plant to plant depending upon their availability. Sometimes more than one part of the species is edible like both leaves and fruits are edible in Spondias pinnata, Luffa acutangula; seeds and young leaves are edible in Lathyrus sativus; young leaves, pods and flowers are edible in Bauhinia purpurea, B. variegata; fruits, young leaf tendrils and flowers are eaten in Cucurbita moschata; leaves and tubers are edible in Manihot esculenta; tubers, stem and leaves are edible in Colocasia antiquorum. 


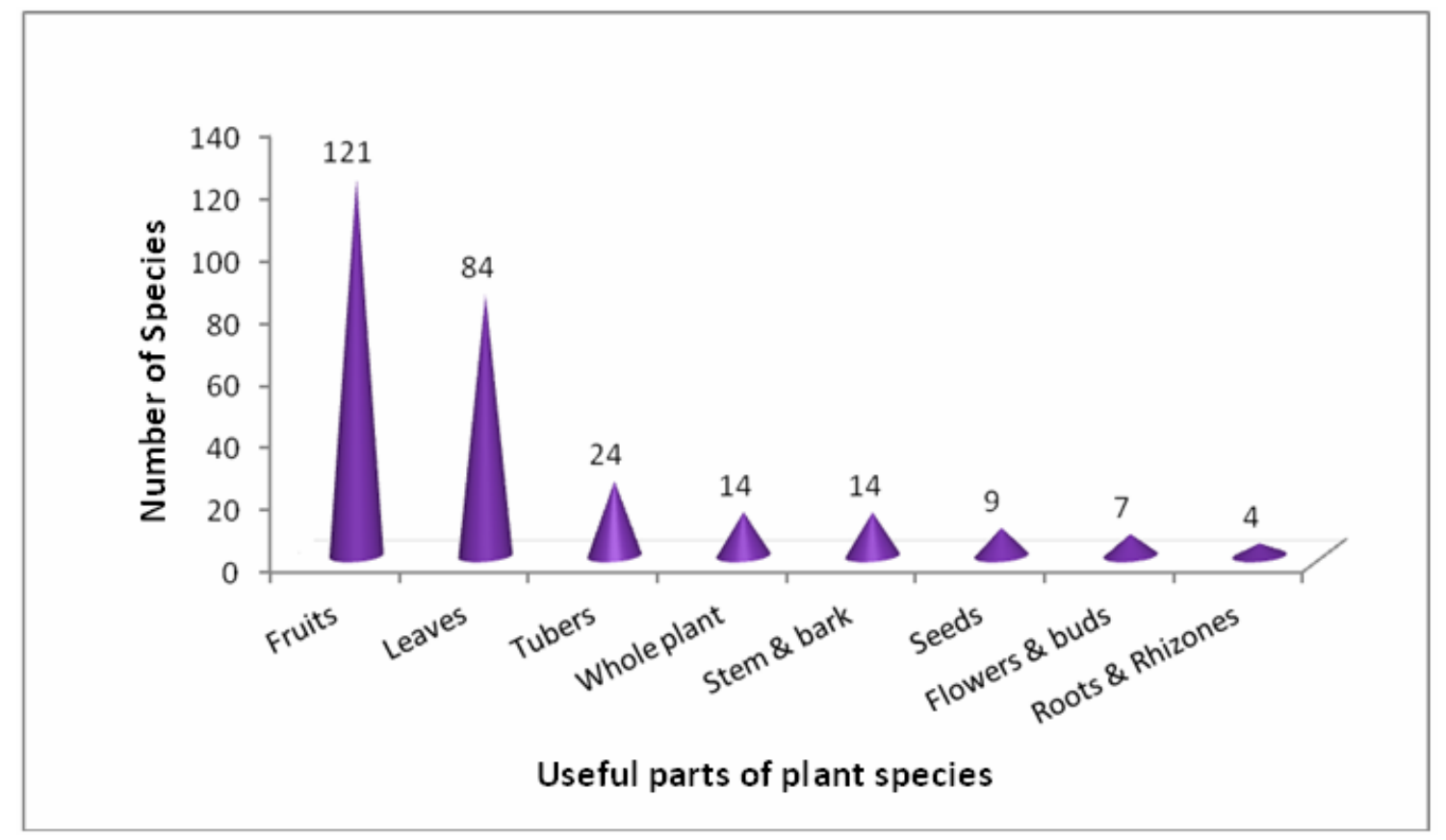

Figure 2. Useful part wise analysis.

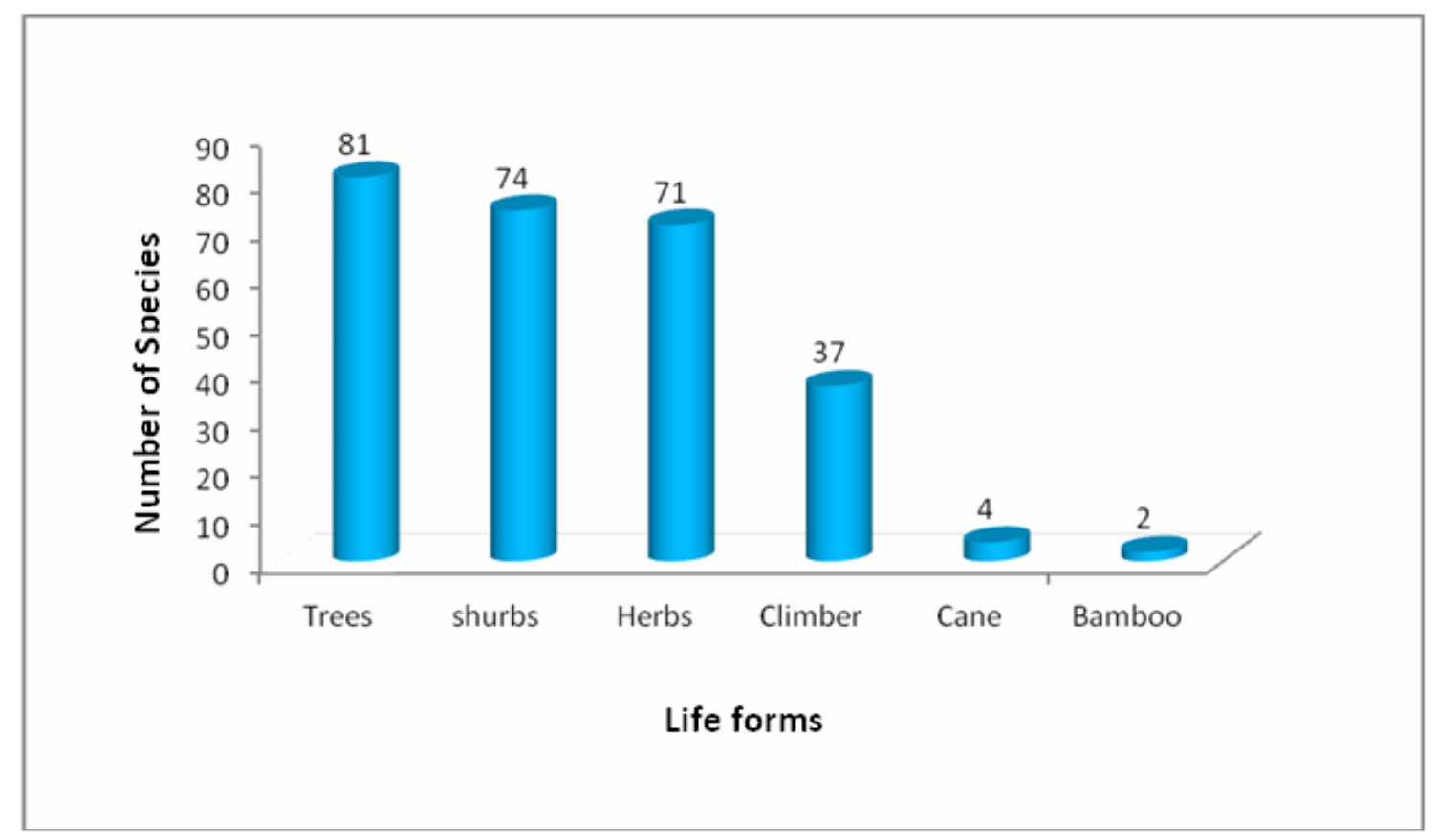

Figure 3. Life form-wise Ethno botanical analysis.

\section{Conclusion}

The discussions with local people revealed that the wild edibles were being used as common household foods and make a substantial contribution to food security of the tribes of the region. Therefore necessary steps should be taken to assess the nutritional value of these plant species/parts to serve as a direct or indirect 
source of food to the local people (Tiwari et al., 2010). There is ample scope for improving the growth forms of wild edible species by using latest agronomic research and experimental cytogenetical studies (Yesodharan and Sujana, 2007). It was commonly noticed during survey that many of the wild food may not be available in plenty as of now due to various reasons mainly over exploitation, habitat destruction, forest fires, encroachments and invasion of exotic plant species. Wherever possible, efforts should be made to bring some of them under cultivation in order to maintain regular supply. Therefore awareness must be created among the indigenous communities stressing the need of conserving rich biodiversity, especially plants of ethnobotanical importance. The Government agencies must support the conservation measures of biodiversity by the indigenous groups. The conservation policies should be based on the recognition of indigenous people's right to conservation. The effective system of community right and benefit sharing mechanism are needed at local level, which will provide incentives for indigenous people for managing, conserving and using biological resources in sustainable manner.

\section{Conflict of interest statement}

Authors declare that they have no conflict of interests.

\section{References}

Arora, R. K. Native food plants of the north eastern tribals. In: Jain, S. K. (Ed.). Glimpse of Indian Ethnobotany. Calcutta: Oxford I. B. H., 1981. p. 91-106.

Goswami, P.; Soki, D.; Jaishi, A.; Das, M.; Sarma, H. N. Traditional healthcare practices among the Tagin Tribe of Arunachal Pradesh. Indian Journal of Traditional Knowledge, v. 8, no. 1, p. 127-130, 2009. Available from: <http://nopr.niscair.res.in/bitstream/123456789/ 2984/1/IJTK 8(1) 127-130.pdf>. Accessed in: Jan. 22, 2016.

Haridasn, K.; Bhuyan, L. R.; Deori, M. L. Wild edible plants of Arunachal Pradesh. Arunachal Forest News, v. 8, no. 2, p. 1-8, 1990.
Hegde, S. N. Conservation of North East Flora. Arunachal Forest News, v. 18, no. 1/2, p. 5-26, 2000.

Khongsai, M.; Saikia, S. P.; Kayang, H. Ethnomedicinal plants used by different tribes of Arunachal Pradesh. Indian Journal of Traditional Knowledge, v. 10, no. 3, p. 541546, 2011. Available from: <http://nopr.niscair.res.in/bitstream/123456789/ 12036/1/IJTK 10(3) 541-546.pdf>. Accessed in: Jan. 22, 2016.

Pal, G. D. Observation on Ethnobotany of tribes of Subansiri, Arunachal Pradesh. Bulletin of Botanical Survey of India, v. 26, p. 26-37, 1984. Avaialble from: <http://www.nelumbobsi.org/index.php/nlmbo/article/view/74831>. Accessed in : Jan 23, 2016.

Pal, G. D. Observation on lesser known interesting tribal use of plants in Lower Subansiri District, Arunachal Pradesh. Journal of Economic and Taxonomic Botany, Additional Series, v. 10, p. 199-203, 1992.

Rawat, M. S.; Shankar, R.; Singh, V. K. Medicinal plants and some folklore of East and West Siang District, Arunachal Pradesh. Bulletin of Medico-Ethnobotany Research, v. 17, p. 17, 1996.

Rawat, M. S.; Chowdhury, S. Ethnomedicobotany of Arunachal Pradesh (Nishi and Apatani) Tribes. Publishers Bishen Singh Mahendra Pal Singh, 1998.

Shankar, R.; Rawat, M. S. Medicoethnobotany of Arunachal Pradesh (Papumpare, Lower Subansiri, Upper Subansiri and Kurungkumey Districts). New Delhi: Himalayan Publishers, 2008.

Shankar, R.; Deb, S.; Sharma, B. K. Antimalarial plants of Northeast India - an overview. Journal of Ayurveda \& Integrative Medicine, v. 3, no. 1, p. 10-16, 2012. Available from: <http://www.jaim.in/temp/JAyurvedaIntegr Med3110-4791493_131834.pdf $>$. Accessed in: Jan. 22, 2016.

Shankar, R.; Lavekar, G. S.; Deb, S.; Sharma, B. K. Traditional healing practice and folk medicines used by Mishing Community of North East India. Journal of Ayurveda \& Integrative Medicine, v. 3, no. 3, p. 124-129, 2012. Available from: <http://www.jaim.in/temp/JAyurvedaIntegrMed 33124-4898143_133621.pdf >. Accessed in: Jan. 22, 2016.

Tag, H.; Das, A. K. Ethnobotanical notes on the Hill Miri Tribe of Arubachal Pradesh. Indian Journal of Traditional Knowledge, v. 3, no. 1, p. 80-85, 2004. Available from: <http://nopr.niscair.res.in/bitstream/123456789/ 
9339/1/IJTK 3(1) 80-85.pdf>. Accessed in: Jan. 22, 2016.

Tiwari, J. K.; Ballabha, R.; Tiwari, P. Some promising wild edible plants of Srinagar and its adjacent area in Alaknanda Valley of Garhwal Himalaya, India. Journal of American Science, v. 6, no. 4, p. 167-174, 2010. Available from: <http://www.jofamericanscience.org/ journals/am-sci/am0604/24_2477_am0604_167 _174.pdf>. Accessed in: Jan. 22, 2016.
Yesodharan, K.; Sujana, K. A. Wild edible plants traditionally used by the tribes in the Parambikulam Wildlife Sanctuary, Kerala, India. Natural Product Radiance, v. 6, no. 1, p. 74-80, 2007. Available from: $<$ http://nopr.niscair.res.in/bitstream/123456789/ 7837/1/NPR 6(1) 74-80.pdf $>$. Accessed in: Jan. 22, 2016.

License information: This is an open-access article distributed under the terms of the Creative Commons Attribution License, which permits unrestricted use, distribution, and reproduction in any medium, provided the original work is properly cited. 